\title{
Theoretical analysis of steady state operating forces in control valves
}

\author{
Hubballi Basavaraj ${ }^{1 *}$ and Sondur Vilas ${ }^{2}$ \\ ${ }^{1}$ Visvesavraya Technological University, Jain College of Engineering Belagavi, India \\ ${ }^{2}$ Visvesvaraya Technological University, Belagavi, India
}

\begin{abstract}
The controlling components, such as valves are used to regulate controlled fluid power. It is not always possible to calculate valve forces accurately, and with some types of valves even the existence of certain types of forces cannot be predicted with certainty. In many cases, however, the analysis can be made fairly completely and accurately. The assumption of steady state conditions is valid for the valve alone, but transient effects in the rest of the system may be large. These effects are particularly important with regard to the instability of valves, where the system may react on the valve in such a way as to make it squeal or oscillate, sometimes with large amplitude. The origin of the steady state flow force understood from a brief qualitative explanation. The following paper will summarize much of what is known about valve forces in the spool type controlling element.
\end{abstract}

\section{Introduction}

In order to determine the transient or dynamic charcteristic of a hydraulic control circuit, the complete charcteristics of each component must be known. The basic information is required for designing of hydraulic control components but also for the proper selection of the right components and control circuits which will result in optimum overall system performance. Control valves of the types with which are consist of arrangements of orifices, variable and fixed, which are so connected that they can vary the restrictions which they offer to the flow of liquid from sources to load, in the desired fashion, as the moving member of the valve is positioned by some eternal agent.

The hydraulic components must closely match the elements preceding them in the chain of command and then must perform the assigned output function precisely during both steady-state and transient operation. In order to make the proper selection, there must be complete familiarity with the various types of hydraulic control components as well as their steady-state and dynamic characteristics. Every component type has its own particular features and limitations, such as hysteresis, dead band, threshold signal level and nonlinearities.

\footnotetext{
*Corresponding author: bvhubliabhi@gmail.com
} 


\section{Literature Review}

J F.blackburn, et al. [1] shown the effect of different spool designs on the forces with some experimental work. The operating member of a control valve-spool is subjected to a number of different forces while it is in operation. These forces have various origins and various characteristics, and it is necessary to know their magnitudes and natures in order to design for successful operation of the valve. In many cases, these forces are large enough to cause erratic operation, or even to prevent operation completely, if suitable precautions are not taken.

R. Amirante, et al. [2] analyze the behaviour of the flow force peak values in the initial spool opening of an open center directional control valve and to establish a connection to the behaviour of a closed center valve. In order to evaluate the different behaviours of the valve, this analysis will be conducted at different flow rates. R. Amirante, et al.[3] analysis made to evaluate the valve fluid dynamic performance, exploiting computational fluid dynamics (CFD) techniques, in order to give the reliable indications needed to define the valve design criteria and avoid expensive experimental tests. M. Batoli, [4] studied the position of flow force peak value on a closed center directional control valve. M. Borghi at al. [5] provided the fluid dynamic similarity based procedure to estimate analytically the flow force peak value at different pressure drops and flow rates. In many literatures analyzed the effects of different spool edge shapes produce on the flow force profile during the axial spool movement [6-9].

\section{Methodology}

The main objective of this paper is about the discussion of forces acts on the directional control valve spool. It is analyzed that, maximum force is greatly decreased by the use of modified construction (Half round baffle). Further substantial reductions can be made by minor adjustments of the dimensional parameters and by improved workmanship.

The operating member of a control valve-the spool is subjected to a number of different forces while it is in operation. Alomg with the force supplied by the valve-operating device, but in addition other forces may either aid or oppose the operating force. These forces have their magnitudes and natures in order to design for successful operation of the valve. In many cases, these forces are large enough to cause erratic operation, or even prevent operation completely, if suitable precautions are not taken.

The forces which act upon a valve spool may be classified according to their direction of action as peripheral, lateral and axial. The first class is usually negligibly small. Lateral forces are not directly important since they act normal to the direction of motion of the spool, but in practice they may be very large so that the friction of the spool against the sleeve becomes excessively great. This friction may be so large in serious cases that the spool becomes immovable, hence the name "hydraulic lock". Axial forces are naturally important since they directly affect the operation of the valve. They are often considerably greater than the inertial and frictional forces, and hence, they directly determine the design of the valve-operating device. In addition they often cause the valve to oscillate or "sing" and this tendency alone may make an otherwise good valve useless valve for a particular application.

\subsection{Lateral Forces-Friction and Hydraulic Lock}

Valve sticking or excessive friction, although usually greatly aggravated by the presence of dirt in the oil, frequently occurs in spite of all precautions to exclude dirt. It has long been 
known that the condition is worst with plane pistons and that it can be greatly alleviated by adding peripheral grooves around the piston lands. The standard explanation for the effect of the grooves is that they act to equalize the pressure distribution around the land. It is of interest to develop a quantitative theory of the pressure distribution and the resulting land force for various land configurations.

The following assumptions will be made that fully developed laminar flow is present throughout the length of the flow path.

1) Cylinder bore is perfectly straight but the spool land is not.

2) Inertial and gravitational effects will also be neglected.

3) All the flow is parallel to the axis and that the peripheral component of flow is negligibly small.

First consider the case of a truly cylindrical spool in a true bore, with the axes of the spool and bore parallel but non-coincident. Consider the flows $\mathrm{dq}_{1}$ and $\mathrm{dq}_{2}$ through two elements of the peripheral clearance space at the top and bottom of the spool, the spool being displaced upward as in Fig. 1., each element has a peripheral width, $d z=a d \theta$, normal to the plane of the figure. Since for each elementary conduit the cross-sectional area, $\mathrm{y}_{1} \mathrm{dz}$ or $y_{2} d z$, is constant over the length, 1 , of the spool land, the pressure gradient $d p / d x=\left(P_{1}\right.$ $\left.\mathrm{P}_{2}\right) / 1=$ constant for both conduits.

Thus the curve of pressure versus $\mathrm{x}$ is a staright line between the points $\mathrm{P}_{1}$ and $\mathrm{P}_{2}$ at the ends of the spool for each of the two conduits.

The downward force on the spool is

$$
d f_{1}=\int_{0}^{l} p d z d x=\frac{P_{1}+P_{2}}{2} l d z
$$

The upward force, $\mathrm{df}_{2}$, is exactly the same; the $\mathrm{df}$ 's balance by pairs all around the spool and the net lateral force is zero for any case in which the surface of the spool is parallel with the adjacent wall of the cylinder.

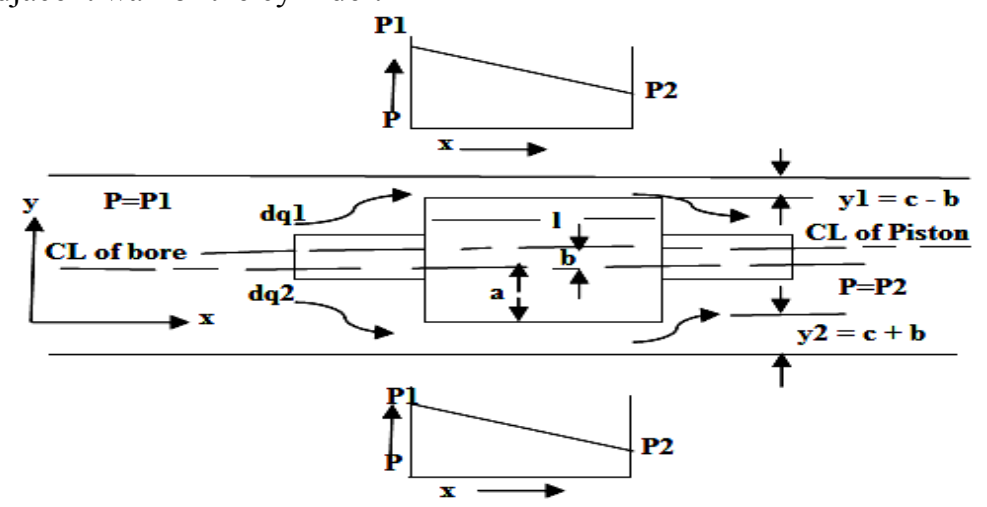

Fig. 1. Cylindrical decentered piston

The net force is also zero for any configuration possessing axial symmetry, but this is not necessarily true for decentered spools, where the axial symmetry is destroyed. The simplest case of this type is that of a decentered conical shown in Fig. 2. Here the crosssectional area and, therefore the pressure gradient are no longer constant over the length of the spool, and the force per unit peripheral width, dz, must be found by a double integration.

The resulting formulas are:

For an elementary length, $\mathrm{dx}$, of the conduit of width $\mathrm{dz}$ and height $\mathrm{y}$,

$$
\frac{d p}{d x}=-\frac{12 \mu d q}{y^{3} d z}
$$


where $\mu$ is the absolute viscosity and $\mathrm{dq} / \mathrm{dz}$ is the flow through a conduit of width $\mathrm{dz}$. With integration and insertion of the boundary conditions,

When $\mathrm{x}=0, \mathrm{y}=\mathrm{C}_{1}$, and $\mathrm{p}=\mathrm{P}_{1}$

And when $\mathrm{x}=1, \mathrm{y}=\mathrm{C}_{1}+\mathrm{t}$, and $\mathrm{p}=\mathrm{P}_{2}=\mathrm{P}_{1}-\Delta \mathrm{P}$

$$
\text { We get } p=P_{1}-\frac{\Delta p\left(C_{1}+t\right)^{2}}{t\left(2 C_{1}+t\right)}\left(1-\frac{C_{1}^{2}}{y^{2}}\right)
$$

where $t$ is the radial taper of the piston. Thus, the variations of pressure with ( $y$ and hence with) $\mathrm{x}$ is parabolic.

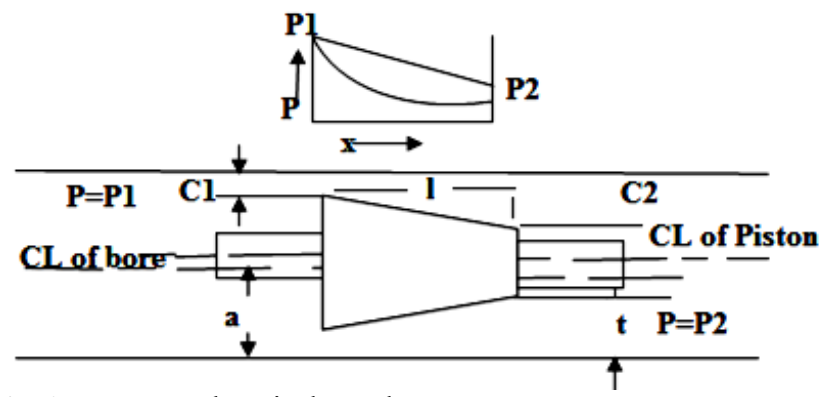

Fig. 2. Decentered conical spool

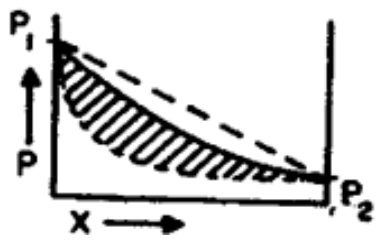

The actual deviation of this parabolic pressure curve from the linear curve of a uniform conduit depends essentially upon the taper and upon the closeness of approach of the large end to the cylinder wall. For the upper duct of fig, the large end approaches closely, the pressure gradient near the large end is high, and the $\mathrm{p}-\mathrm{x}$ curve drops rapidly from the $\mathrm{P}_{1}$ point. For the lower duct, the ratio of the y's at the upstream and downstream ends is much nearer unity, and the departure from linearity is much less.

The radial force on an elementary area, $\mathrm{dx} d \mathrm{z}$, of the piston will be $\mathrm{p} \mathrm{dx} \mathrm{dz}$. Integration gives the total radial force on the elementary strip of width $\mathrm{dz}$ as

$$
d f=P_{1}-\left(\frac{\left(C_{1}+t\right) \Delta P}{\left(2 C_{1}+t\right)}\right) l d z
$$

The total lateral force $\mathrm{F}$ on the spool is obtained by integrating $\mathrm{df}$ around the periphery, when account is taken of the fact that only the component of df parallel to the displacement $\mathrm{b}$ is of-interest since the normal components will cancel because of the symmetry of the configuration. Thus, if $\mathrm{df}$ is the component of $\mathrm{df}$ parallel to $\mathrm{b}$,

$$
d F=-l a\left(P_{1}-\Delta P \frac{C+t+b \cos \theta}{2 C+t+2 b \cos \theta}\right) \cos \theta
$$

where $\mathrm{C}$ is the radial clearance at the large end with the spool centered. Integration with respect to $\theta$ from 0 to $2 \pi$ gives the total side-thrust on the piston:

$$
F=\frac{\pi a t \Delta p}{2 b}\left(1-\frac{2 C+t}{\sqrt{(2 C+t)^{2}-4 b^{2}}}\right)
$$

Since the second term in the parentheses is greater than 1 , the force will be negative, that is, away from the larger opening, and is therefore a decentring force. Thus the equilibrium of a centered tapered spool with the higher pressure applied to the larger end is unstable, and the spool will be forced into contact with the wall. Conversely, if the higher pressure is applied to the smaller end, the spool will center itself in the bore.

Calculations have been made for one land of a very small pilot spool which stuck persistently. The resulting curves for $F$ versus $b$ are plotted in Fig. 3. 


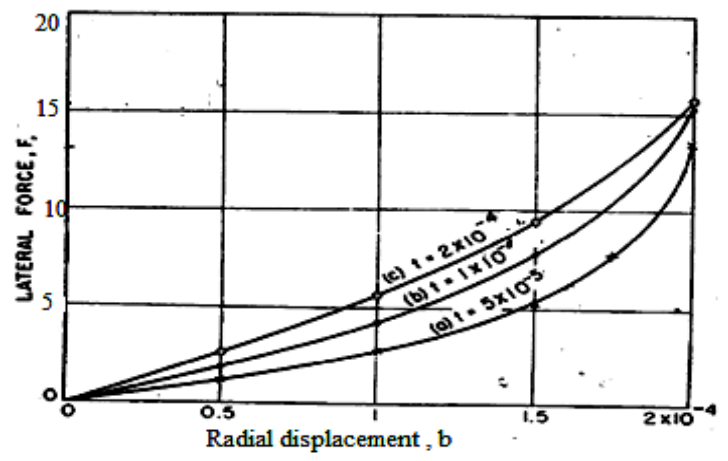

Fig. 3. Lateral force versus displacement for a small spool land

The values of the various quantities involved are: Axial length, $1=2.4 \mathrm{~mm}$, radius, $\mathrm{a}=$ $1.6 \mathrm{~mm}$, radial clearance at larger end, $\mathrm{C}=0.0051 \mathrm{~mm}$, Pressure drop, $\Delta \mathrm{p}=1000 \mathrm{psi}$, Radial taper, $\mathrm{t}=0.002 \mathrm{~mm}$. for curve $(\mathrm{a}),=0.0025 \mathrm{~mm}$, for curve $(\mathrm{b}),=0.005 \mathrm{~mm}$, for curve (c)

From Fig. 3, it can be seen that the peripheral effects of varying the amount of tapers are: (1) the rate of increase of decentering force for small displacements from center decreases rapidly with decreasing taper, and (2) the force holding the spool against the wall (when $\mathrm{b}=0.0051 \mathrm{~mm}$, in the present case) changes comparatively little.

\subsection{Axial Forces}

The axial forces that operate on a valve spool include the inertial force, which is readily calculated and is usually small, the frictional forces, which are not accurately calculable but which should also be small in a properly designed and properly applied valve, and various forces which owe their existence to the fact that fluid is flowing through the valve and exerting force on the spool. Knowledge of these flows-produced forces is still incomplete and every new basic valve design presents new problems, but for certain common types of valves, the principal forces are known, and their magnitudes can be calculated with good accuracy [10]. 

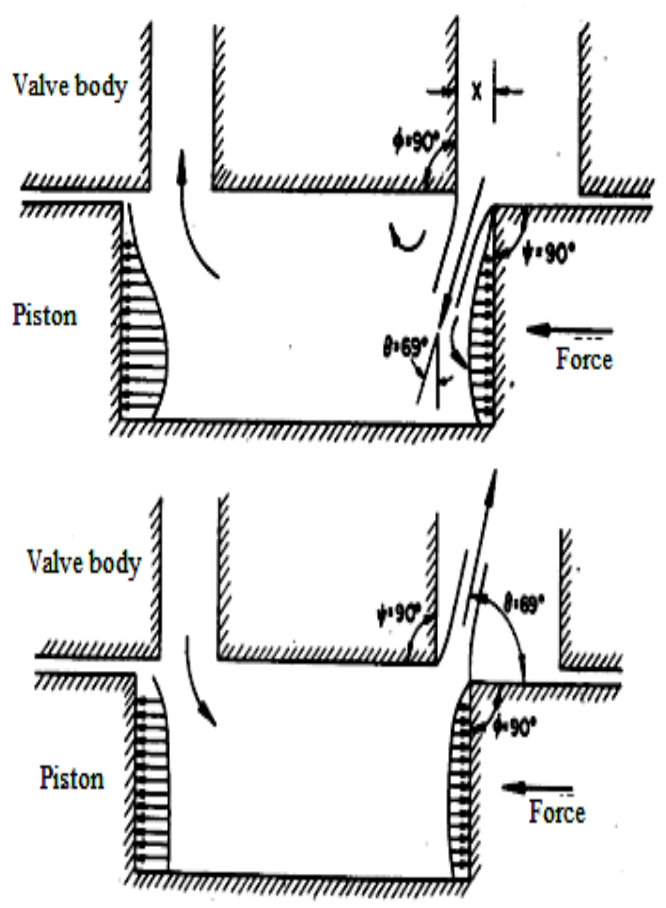

(a) Jet Entering Piston Chamber (b) Jet leaving piston chamber

Fig. 4. Origin of the steady state flow force

In Fig. 4, which represents one metering orifice of a spool valve, with the upstream and downstream chambers and the connecting ducts, the cross-sectional area of the stream near the orifice is much smaller than it is everywhere else, and therefore, the velocity of flow is small except near the orifice edges.

A quantitative solution of this case can be found as follows. Assuming:

1) The fluid is nonviscous and incompressible.

2) The peripheral width of the orifice is large compared with its axial length so that the flow can be considered to be two-dimensional.

3) The flow is assumed to be quasi-irrotational in a region immediately upstream from the orifice.

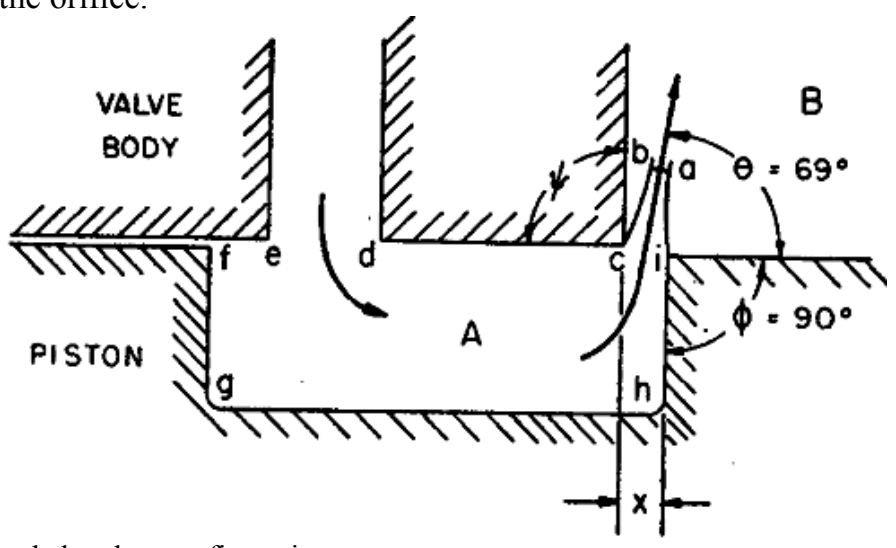

Fig. 5. Square-land chamber configuration 
With the assumptions that the flow is two-dimensional, irrotational, nonviscous, and incompressible, the solution of the flow pattern in the region upstream from the orifice becomes a solution of Laplace's equation for the configuration shown in Fig. 5. this solution has been found by vonmises, and it is found that, for a square-land valve when the valve opening, $\mathrm{x}$, is small compared with the other dimensions of the upstream chamber, the angle $\theta$, which the axis of the stream makes with the spool axis is $69^{\circ}$, this value has been experimentally verified both on actual valves and on a two-dimensional valve model with glass sides [1]. For lands with angles other than 90, the value of $\theta$ can also be found either experimentally or theoretically. When $\theta$ is known, the axial force on the piston can be derived in the following manner.

The axial force on the spool equals the axial component of the net rate of efflux of momentum through the boundary a-b-c-d-e-f-g-h-i-a in Fig. 5, where a-b is the vena contracta of the jet. In an actual valve, the area of $a-b$ is much smaller than that of $d-e$ where the fluid enters the upstream chamber, A. Since the velocities are inversely proportional to the areas, the influx of momentum through d-e is negligible small compared with the efflux at $a-b$, which is equal to $Q U \rho$, where $Q=$ total rate of flow, $U=$ velocity of jet at vena contracta

$\rho=$ density of fluid.

By Bernoulli's equation, $\quad u=\sqrt{ }(2 \Delta p) / \rho$

And the net axial force is:

$$
\mathrm{F}_{\mathrm{AB}}=\mathrm{F}_{\mathrm{fg}}-\mathrm{F}_{\mathrm{hi}}=\mathrm{QU} \rho \cos \theta
$$

This equation can be transformed into the more useful form as:

$$
\begin{aligned}
& \qquad \mathrm{F}_{\mathrm{AB}}=\mathrm{C}_{\mathrm{q}} \mathrm{wx} \Delta \mathrm{p} \cos \theta \\
& \text { where } \mathrm{C}_{\mathrm{q}}=\text { coefficient of discharge }=\frac{Q}{w x \sqrt{(2 \Delta p / \rho)}} \\
& \mathrm{w}=\text { peripheral width of orifice, } \mathrm{x}=\text { axial length of orifice, } \Delta \mathrm{p}=\text { pressure difference } \\
& \text { between chamber A and chamber } \mathrm{B} \text {. }
\end{aligned}
$$

Since $\theta$ is always less than $90^{\circ}$ for valves of the type under discussion, $\mathrm{F}_{\mathrm{AB}}$ of Eq.8. is always positive and tends to close the valve. If the direction of flow is reversed so that the jet flows into chamber $A$ instead of out of it, the equation is still valid, and if the jet angle $\theta$ is the same for both cases, the force will be the same. It should be noted that for Fig. 5, the jet angle depends upon the angle $\phi$ but is independent of $\psi$; for the reversed flow, the opposite is true. In either case, it is the configuration of the upstream chamber that determines the force. Eq. 7. and 8 apply only to an ideal valve with perfectly sharp corners and zero radial clearance. Since such an ideal valve cannot be built, it is important to investigate the effects of these imperfections.

For an orifice with radial clearance, $\mathrm{Cr}$, as shown in Fig. 6. The Eq.8., becomes:

$$
F_{A B}=2 C_{q} w \Delta p \sqrt{x^{2}+C_{r}^{2} \cos \theta}
$$




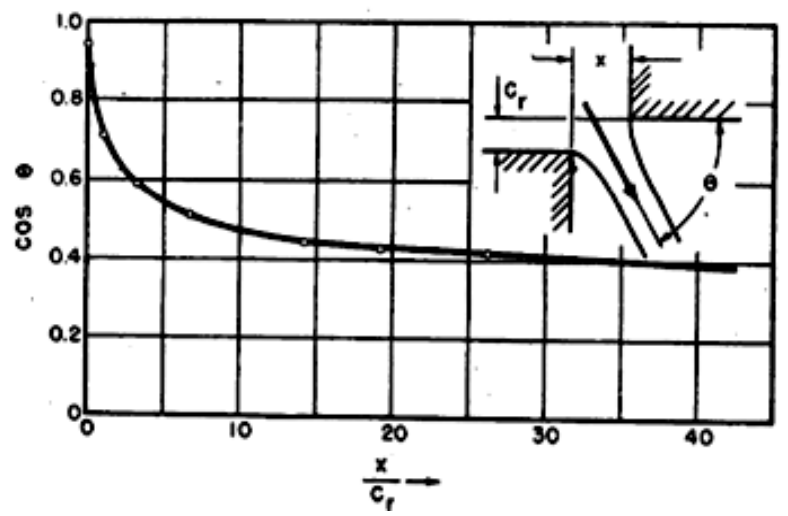

Fig. 6. Effect of radial clearance on $\cos \theta[1]$

Here, $\cos \theta$ is no longer a constant but varies with the quantity $\mathrm{x} / \mathrm{C}_{\mathrm{r}}$, as shown by the curve. If the net force on the spool is plotted against the displacement for a constant value of pressure drop. The curve for an ideal valve is shown dashed in Fig. 7. It can be seen that that the effect of radial clearance is to make the curve rise abnormally rapidly for small openings, after which it sags downward and approaches the ideal curve asymptotically.

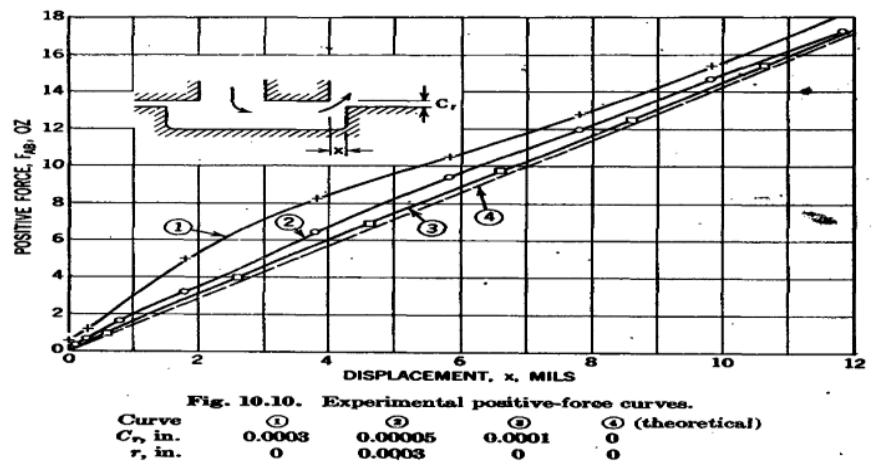

Fig. 7. Experimental positive-force curves [1]

The effect of finite radius of the land edges is difficult to calculate theoretically but is very similar to that of radius clearance. Qualitatively, the variation of $\theta$ can be visualized with reference to Fig. 8.

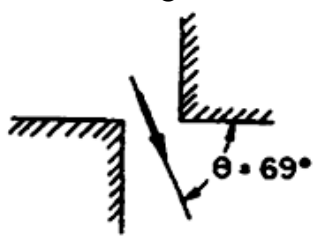

(a)

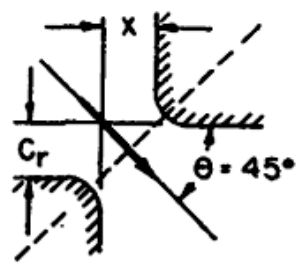

(b)

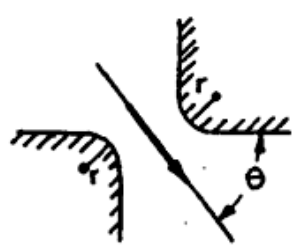

(c)

Fig. 8. Land Configurations

In Fig. 8(a), the ideal case of zero radial clearance and sharp land edges is represented. The figure is skew-symmetrical about the center of the opening; it is the skewness of the symmetry that causes the axis of the jet to deviate from the vertical and it is this deviation and the resulting x-component of momentum that gives rise to the axial force on the spool. 
Fig. 8(b) represents a valve with the axial displacement, $\mathrm{x}$, set equal to the radial clearance, $\mathrm{C}_{\mathrm{r}}$. The figure is symmetrical about the two $45^{\circ}$ lines, and the jet angle, $\theta$, is $45^{\circ}$ because symmetry. This is true also for any value of radius $r$ of the land edges is long as the r's are the same for both edges. If the valve opening, $\mathrm{x}$ is varied, however, the symmetry will be destroyed, and $\theta$ will approach $69^{\circ}$; for small values of $\mathrm{x}$, the jet will approach parallelism with the axis, and the force will be greater than that for an ideal valve.

In a valve with zero radial clearance but finite curvature of the land edges, as in Fig. 8 (c), the symmetry is skewed as for the ideal valve but not to such a great extent; the angle $\theta$ is less than $69^{\circ}$; the piston force is greater than for an ideal valve; and $\theta$ varies with $\mathrm{x}$. For large values of $\mathrm{x}, \theta$ approaches $69^{\circ}$; for negative values of $\mathrm{x}, \theta$ approaches 0 as $\mathrm{x}$ approaches $-r$. In an actual valve in which neither $C_{r}$ nor $r$ is zero, $\theta$ approaches zero at small or negative values of $x$, and the slope of the curve of force versus $x$ increases at most by a factor of $1 / 0.36=2.78$. This is not particularly serious, of course, since in most cases the curve drops off and approaches that for an ideal valve before the actual value of the force is very large; that is, the phenomenon occurs essentially only for small values of $\mathrm{x}$.

In an actual four-way vale, there are two identical orifices in serious and, therefore, there is twice the force on the piston, equation 9 then becomes

$$
F_{T}=2 F_{A B}=2 Q \sqrt{\rho P_{v}} \cos \theta
$$

The radial component of the force on the spool can be easily cancelled by the simple scheme of dividing the port peripherally into two or more equal parts equally spaced around the sleeve. The above analysis of the steady state flow force on a spool valve is assumed to be accurate and adequate for practical application, since it has been verified by a large amount of experimental investigations and operating experience [1]. In so far as the configurations of the metering orifices are similar, this method can be applied to valves of other constructions.

\subsection{Flow-Force Compensation}

Steady state flow forces of the type discussed above have been recognized for years, and various designs have been suggested for reducing or eliminating them. One which works excellently for the valve shown in Fig. 9. In the valve construction to be described, the axial component of efflux of momentum from the piston chamber is made greater than the influx so that a negative (opening) force is developed which $\mathrm{h}$ is proportional to $\mathrm{x}$ for a given pressure drop, and this negative force is balanced by the positive force generated at a conventional square-land orifice. It should perhaps be noted that this method can be used to give a zero-force port, but since the special port construction is somewhat more difficult to manufacture than a conventional square port and since it may lead to the transient instability, the balancing scheme is preferable.

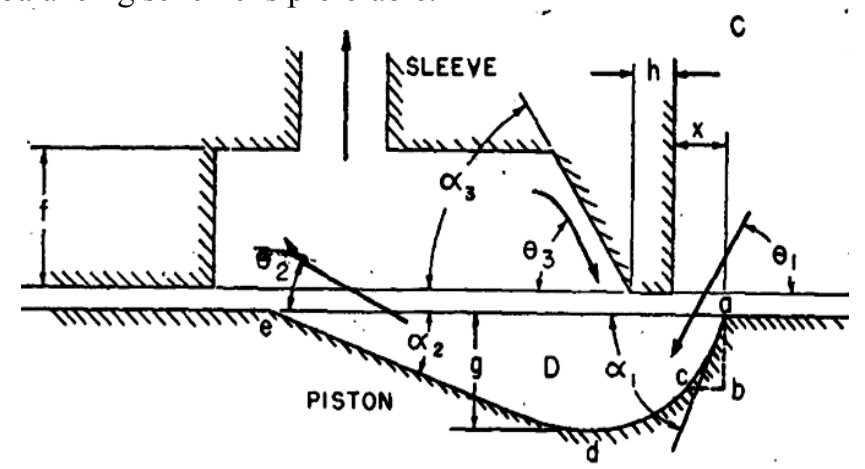

Fig. 9. Negative Force port 
The construction of the negative-force port is shown schematically in Fig.9. It differes from a conventional port in two ways; the chamber in the spool is shaped some what like a turbine bucket, and the sleeve is cut to form an extension of the downstream chamber. Here, the jet through the orifice enters the spool chamber at an angle of $\theta_{1}$, is reflected from the chamber wall near $d$, and leaves at an angle of $\theta_{2}$. If the cross section of the flow were constant and the frictional force negligible, the resulting force on the spool would be

$$
F_{C D}=Q U \rho\left(\cos \theta_{1} \cos \theta_{2}\right)
$$

Thus, the negative force of Eq. 11 may easily become a negative force, since $\cos \theta_{2}$ is ordinarily considerably larger than $\cos \theta_{1}$. Furthermore, an eddy is produced which moves clockwise, and an additional flow enters the piston chamber at an angle of $\theta_{3}$, which increases the negative force still more. The total resultant force on the piston is thus rather complicated. It depends upon numerous variables such as $\alpha_{1}, \alpha_{2}, \alpha_{3}, \rho, \mu, \omega$ and d, where $\rho$ is the density and $\mu$ the viscosity of the fluid, $\omega$ the peripheral width of the port, and $d$ the piston diameter. A theoretical derivation of the force would be practically impossible.

The port width, w, occupies only a portion of the circumference of the spool in many valves. Although it is usually large compared with $\mathrm{x}$ so that the flow can be treated as twodimensional at the orifice, it is small compared with the dimensions of the chambers. When the incomplete jet of fluid hits the spool near the point $d$ and is reflected upward, it tends to spread out normal to the plane of the figure, and the condition of constancy of cross section assumed in Eq. 11 no longer holds. The actual exit angle, $\theta_{2}$, thus tends to be greater than $\alpha_{2}$, and the jet tends to separate from the piston surface. If $\alpha_{1}=\theta_{1}$, the angle of incidence is zero, and the negative force is a maximum; $\alpha_{1}$ should normally be made equal to $69^{\circ}$, the influx angle for a square land. It is found, however, that a square step such as a-b-c in Fig.9 has little effect on the force if it is not too deep, and it is sometimes desirable for manufacturing reasons that such a step be provided. In the case for which $\mathrm{w}=360^{\circ}$, there would be no lateral spreading.

\subsection{Modified Spool Design (Half round baffle type) for Flow-Force compensation}

One possible measure of the effect of the modified construction would be the ratio of the maximum forces on the spool of a conventional and a modified valve. It is possible that, this maximum force is greatly decreased by the use of modified construction. Further substantial reductions can be made by minor adjustments of the dimensional parameters and by improved workmanship. A sketch of a four-way valve of this construction is given in Fig. 10.

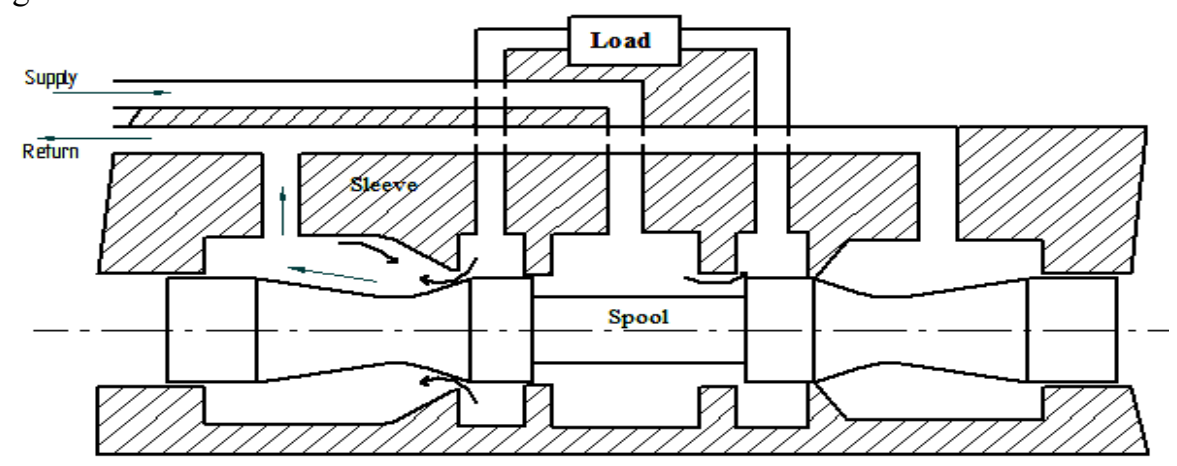

Fig.10. Four-way valve with force compensation (Half round baffle) 
In practice, the force compensation is excellent over most of the range valve openings, and the nonlinearity near the origin. In addition, the above design is much easier to manufacture because the dimensions are not so critical, and the compensation appears to be relatively immune to wear of the metering edges and therefore will change little as the valve ages. Finally, the most attractive feature of this design is that it allows the degree of compensation to be adjusted; if the valve is somewhat overcompensated; the half round baffles can be rotated so as to permit part of the incoming jet to slip by and hit the opposite side of the valve, thereby reducing the compensation. It is easy to find an angle at which the compensation ever deteriorates because of wear or other causes, the baffles can easily be readjusted. This method has been thoroughly tested by many authors with the different geometries in the field.

The method of flow-force compensation that has been outlined is good in theory, and should be excellent in practice for long-stroke valves. Even if the compensation is initially excellent, the inevitable erosion of the land corners by dirt in the oil acts to make it less effective after the valve has been in service for a while, and this tends to restrict its application to short-life or expendable devices. Furthermore, even if wear and life are not important factors, the radical nonlinearity of the relationship between the valve force and displacement at small openings makes it difficult to use the valve in many highperformance systems.

The principal disadvantages of this design are that it is usually applicable only to a very restricted range of operating conditions, that it is very difficult to design and mechanize, and particularly, it is very desirable to have as strong an actuator as possible in order to have some margin of force to take care of excessive friction or other to have some margin of force to take care of excessive friction and other sponging effects. Also, even if the force available from the actuator cannot be increased, it is still very desirable to have the actuator as stiff as possible on the interests of valve stability.

\section{Conclusions}

Most of the designs that have been proposed for the compensation of flow forces fall into one or the other of two categories: either the incoming jet is deflected in such a way that it carries out of the piston chamber the momentum that it brought in (or more), or else this momentum is caught and transferred to the sleeve by some sort of vane or other member which projects into the spool chamber. In concluding this, it is pertinent to point out that compensation of flow forces is not always necessary, or even desirable. If plenty of force is available to actuate the valve, compensation is unnecessary and merely adds complication and expense.

It is not always possible to calculate valve forces accurately, and with some types of valve even the existence of certain types of forces cannot be predicted with certainty. In many cases, however, the analysis can be made fairly completely and accurately. The most of derivations will apply primarily to spool valves, but the formulas will apply to other construction as well, although they may have to be modified appropriately. A three dimensional analysis of a spool type control valve is essential to determine correctly the flow force and the flow rate values, especially for the designs and avoids expensive tests.

\section{References}

1. J F.blackburn, et al, "Fluid Power Control", John Wiley and Sons Inc, Newyork London,(1960) 
2. R. Amirante, G. Del Vescovo and A. Lippolis "Flow forces analysis of an open center hydraulic directional control valve sliding spool", Energy Conversion and Management Elsevier ,47 114-131, (2006)

3. R. Amirante, G. Del Vescovo and A. Lippolis "Evaluation of the Flow forces on an open center directional control valve by means of a computational fluid dynamic analysis", Energy Conversion and Management Elsevier, 47, 1748-1760 (2006)

4. M. Batoli, "Theoretical and experimental analysis of flow forces on a hydraulic directional control valve", Pneumatica: 3, 6-10 (1996)

5. M.Borghi, M.Milani, R.Paoluzzi, "Stationary axial flow force analysis on compensated spool valves", International Journal of Fluid Power; 1, 17-25, (2000)

6. A.Macor, S.Dato, "Flow force reduction in a hydraulic directional control valve by means of a restricted section shaping". In: Proceedings of the 55 Congresso nazionale ATI, Bari- (2000)

7. A.Macor, "Experimental analysis on a directional valve with a flat notch metering section", In: Proceedings of the 57 Congresso Nazionale ATI, Pisa (2002).

8. G.Del Vescovo, A. Lippolis, "Flow forces analysis on an electro piloted hydraulic directional control valve" In: Proceedings of the 3rd International Congress MiniHydro, (2001)

9. G.Del Vescovo, A. Lippolis, "CFD analysis of flow forces on spool valves", In: Proceedings of the First International Conference on Computational Methods in Fluid Power Technology, Melbourne, 26-28, (2003)

10. B V. Hubballi and Dr V B. Sondur, "Directional Control Spool Valve Performance Criteria and Analysis of Flow-Reaction Forces", International Journal of Emerging Technology and Advanced Engineering, Volume 5, 86-93, (2015) 